\title{
Synthesis of Silicon Oxide Nanowires by Chemical Vapor Deposition
}

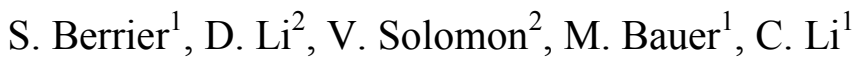 \\ 1. Clarion University, Physics Department, Clarion, PA 16214 \\ 2. Youngstown State University, Engineering Department, Youngstown, OH 44555
}

Nanowires can be fabricated by numerous methods such as sol-gel processing, thermal oxidation, ion implantation, and chemical vapor deposition (CVD) [1]. The CVD method either utilizes a vapor solid (VS) or vapor liquid solid (VLS) growth mechanism. The VLS growth mechanism, a liquid catalyst ( $\mathrm{Au}, \mathrm{Fe}, \mathrm{Ga}$, etc.), absorbs and precipitates the nanowire material. Among the possible catalysts for silica nanowire growth, gallium has been shown to be a low melting point catalyst which effectively produces large scale growth of highly aligned and closely packed silica nanowire bunches. Additionally, the molten Ga catalysts can be precipitated onto the substrate in the same processing step as the silica nanowire synthesis. Some of the other benefits to using $\mathrm{Ga}$ as a catalyst are that $\mathrm{Ga}$ is able to solve $\mathrm{Si}$ at a wide range of temperatures and it does not react with $\mathrm{Si}$ [2].

The CVD experiments used $\mathrm{Ga}_{2} \mathrm{O}_{3}, \mathrm{ZnO}$, graphite powder, and silicon wafer substrates. The graphite serves as a source of carbon monoxide which reduces the gallium oxide to gallium suboxide which is then vaporized and deposited on the substrate as gallium (1) and gallium oxide (s). The liquid gallium then absorbs the silicon from the silicon wafers and precipitates as silicon oxide nanowires [3].

The CVD method of nanowire fabrication was used to synthesize the nanowires within a horizontal tube furnace. The source powder was placed in an alumina boat at the center of the tube furnace with an internal temperature of $1200^{\circ} \mathrm{C}$. A variance in vacuum pressure, on the order of $10^{-1}$ torr, was used. The vapors were deposited onto the silicon wafers extending away from the center of the furnace. These wafers were centered on the exit of the furnace: three outside and three inside, with the wafers placed most inside the furnace heated to the highest temperature and those most outside having the lowest temperature during deposition.

The nanowire deposition on the wafers was affected by the variance in vacuum pressure. In one experiment the pressure settled in the range of $1 \times 10^{-1}$ to $9 \times 10^{-2}$ torr. Upon examination with a scanning electron microscope (SEM), the nanowires formed in this experiment were found on the innermost and next to outermost wafers. Those on the innermost wafer closest to the center of the tube furnace, in the form of densely packed singular wires, were examined by energy dispersive $\mathrm{x}$-ray spectroscopy (EDS) and were primarily composed of silicon and oxygen. The nanowires on the second from the outermost wafer, formed as gourdlike bunches, were also examined by EDS and were composed of gallium and silicon. In another experiment the pressure level was lower, at a level of $4 \times 10^{-1}$ torr. The nanowires at this pressure formed on the wafer second from the inside of the furnace. Analysis by EDS showed that these wires were primarily composed of silicon and oxygen. No deposition indicating the presence of nanowires on the outer wafers was observed in this case.

\section{References}

[1] Cheong, K.Y. and Chiew, Y.L., Nanowires Science and Technology (ed. Nicoleta Lupu), Ch. 7, 402 (2010). 
[2] Pan, Z.W., Dai, Z.R., Ma, C. and Wang, Z.L., Molten gallium as a catalyst for the large scale growth of highly aligned silica nanowires. Journal of the American Chemical Society 124, 1817-1822 (2002). [3] Butt, D.P., Park, Y. and Taylor, T.N., Thermal vaporization and deposition of gallium oxide in hydrogen. Journal of Nuclear Materials 264, 71-77.

[4] Financial support by National Science Foundation (DMR-1229063) is acknowledged.
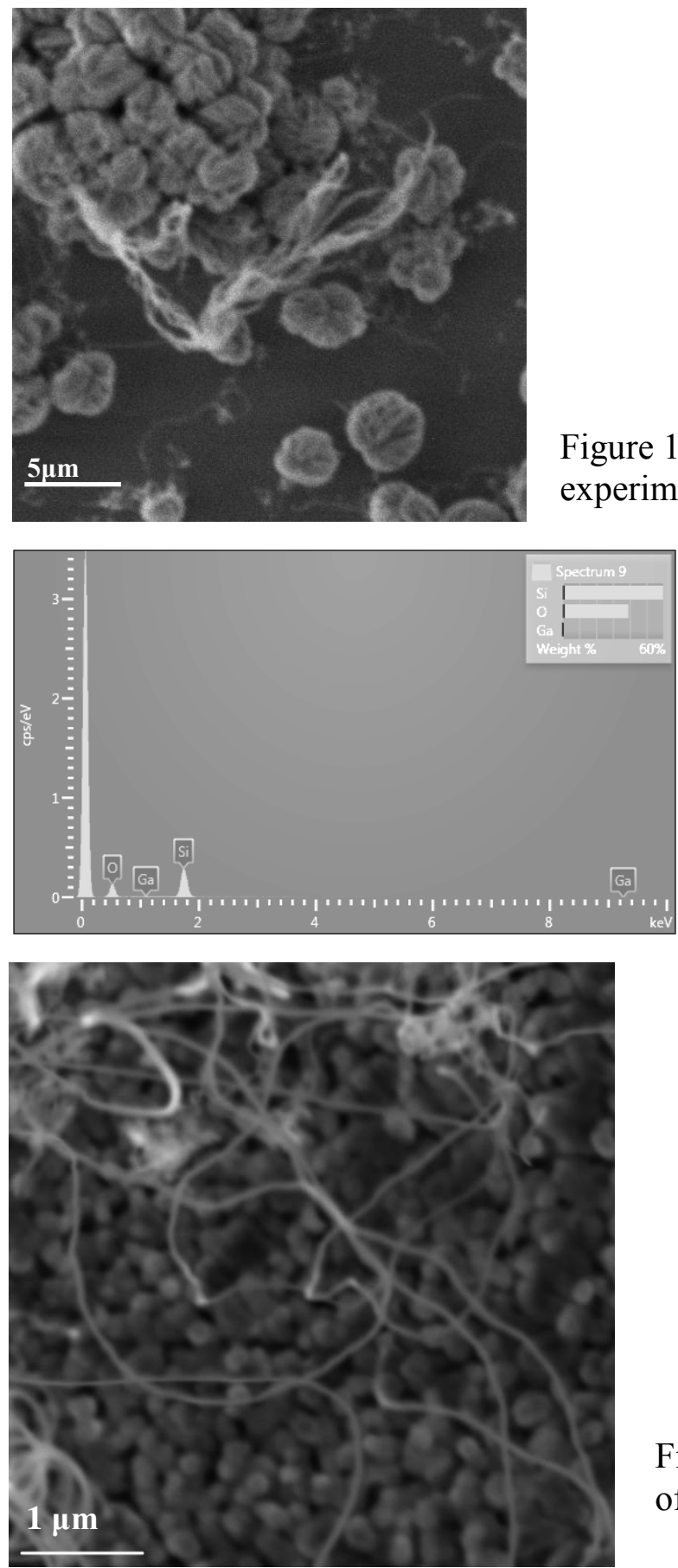

Figure 1: Gourdlike bunches present on outer wafer during experimental run of higher pressure.

Figure 2: EDS spectra for the gourdlike bunches formed around a gallium center.
Figure 3: Singular wires formed at a lower pressure of $4 \times 10^{-1}$ torr. 